\title{
Clinical and demographic characteristics of patients with COVID-19 infection: Statistics from a single hospital in Iran
}

\author{
Majid Samsami ${ }^{\mathrm{a}}$, Elham Mehravaran ${ }^{\mathrm{b}}$, Payam Tabarsi ${ }^{\mathrm{c}}$, Abdolreza Javadi ${ }^{\mathrm{d}}$, Shahram Arsang-Jang ${ }^{\mathrm{e}}$, \\ Alireza Komaki ${ }^{\mathrm{f}}$, Mohammad Taheri ${ }^{\mathrm{g}, *}$ and Soudeh Ghafouri-Fard ${ }^{\mathrm{h}}$ \\ ${ }^{a}$ Cancer Research Center, Shahid Beheshti University of Medical Sciences, Tehran, Iran \\ ${ }^{\mathrm{b}}$ Motamed Cancer Institute, Tehran University of Medical Sciences, Tehran, Iran \\ ${ }^{\mathrm{c}}$ Clinical Tb and Epidemiology Research Center, NRITLD, Shahid Beheshti University of Medical Sciences, Tehran, \\ Iran \\ ${ }^{\mathrm{d}}$ Department of Pathology, Imam Hossein Hospital, Shahid Beheshti University of Medical Sciences, Tehran, Iran \\ ${ }^{\mathrm{e}}$ Department of Biostatistics and Epidemiology, Cancer Gene Therapy Research Center, Faculty of Medicine, Zanjan \\ University of Medical Sciences, Zanjan, Iran \\ ${ }^{\mathrm{f}}$ Neurophysiology Research Center, Hamadan University of Medical Sciences, Hamadan, Iran \\ ${ }^{\mathrm{g}}$ Urogenital Stem Cell Research Center, Shahid Beheshti University of Medical Sciences, Tehran, Iran \\ ${ }^{\mathrm{h}}$ Department of Medical Genetics, Shahid Beheshti University of Medical Sciences, Tehran, Iran
}

\begin{abstract}
Coronavirus disease 2019 (COVID-19) has caused a global pandemic in early 2020. This infectious disorder has a heterogeneous course ranging from asymptomatic disorder to a critical situation needing intensive cares. In the current study, we present a report of affected patients admitted in a single hospital in Iran. Eighty-two hospitalized patients with COVID-19 were assessed. Demographic, clinical, and paraclinical parameters were gathered and statistically analyzed. The median age (IQR) of the patients was $57.32(45.75,70)$ years. At primary evaluation, fever was present in $45.12 \%$ of the affected individuals. The most common clinical symptoms were dyspnea (81.71\%) and cough (65.85\%). Totally, $12(14.63 \%)$ and $14(17.07 \%)$ of patients had low and high WBC counts, respectively. Lymphopenia was detected in $36(43.9 \%)$ of patients, while $6(7.32 \%)$ of patients had lymphocytosis. High levels of Il-6 were detected in 4 (4.88\%) of patients. CRP levels were elevated in $69(84.1 \%)$ of patients. The median (IQR) of hospitalization was $7(5,9)$ days. Totally, 26 patients $(31 \%)$ were hospitalized in ICU. All patients were discharged with good health conditions except for one patient who died. The current study shows the heterogeneous clinical manifestations and paraclinical parameters of COVID-19 patients.
\end{abstract}

Keywords: COVID-19, Iran, demographic data

\section{Introduction}

Coronavirus disease 2019 (COVID-19) resulting from the severe acute respiratory syndrome coronavirus 2 (SARS-CoV-2) has caused a global pandemic in early

*Corresponding author: Mohammad Taheri, Urogenital Stem Cell Research Center, Shahid Beheshti University of Medical Sciences, Tehran, Iran. E-mail: mohammad_823@yahoo.com.
2020 [1]. Iran has been among the first countries experienced epidemic of this viral infection [2]. The clinical manifestations of COVID-19 is quite wide, including asymptomatic infection, minor upper respiratory tract disease, and severe extensive pneumonia leading to respiratory distress and even demise [3-5]. Disease severity is influenced by a number of population specific parameters [6] necessitating recognition of possible risk factors and clinical manifestations in each 
population. A recent meta-analysis of 32 papers reporting the clinical manifestations of COVID-19 in 49504 patients has demonstrated fever, cough and fatigue as the most common symptoms, respectively. Bilateral pneumonia, ground-glass opacity, thrombocytopenia and lymphocytopenia were the most common radiological and paraclinical manifestations [7]. Few studies have analyzed COVID-19 course and mortality-related risk factors in Iranian population. In a single-center study, Ashraf et al. have reported shortness of breath and cough as the most frequent symptoms at the time of admission. Moreover, they reported the presence of fever in $45.2 \%$ of the patients at that time. Death occurred in $4.3 \%$ of their admitted patients [8]. Another single-center study from Iran reported mortality rate of $7.14 \%$ for admitted patients with COVID-19. Moreover, they reported extensive involvement of lungs and remarkable damage to the pulmonary tissue in these patients [9]. In the current study, we report the clinical, paraclinal and demographic data of patients admitted to Nikan hospital, Tehran with the confirmed diagnosis of COVID-19.

\section{Methods}

\subsection{Patients}

In the current retrospective, single-center study, clinical and demographic data of patients admitted to Nikan Hospital, Tehran were obtained through assessment of medical records. Patients were admitted during 23 March till 23 April 2020. Patients with flu-like syndrome or other related symptoms were assessed. Initial diagnosis was performed through physical examination and radiologic pulmonary findings. Diagnosis was confirmed by real time RT-PCR of nasopharyngeal samples. The study protocol was approved by ethics committee of Shahid Beheshti University of Medical Sciences (IR.SBMU.MSP.REC.1399.62). Informed consent was obtained from all patients. A complete paraclinical investigation and chest CT scan were performed for all admitted patients.

\subsection{Statistical methods}

Quantitative variables were described by the median and interquartile range (IQR) values. The Bayesian One sample $t$ test and nonparametric median test were used to compare the parameters with standard values. The one sample proportion test was used to compare proportion of events (Yes category) with 0.5 .
Table 1

Clinical data of admitted patients

\begin{tabular}{llc}
\hline Symptoms and signs & No $n(\%)$ & Yes $n(\%)$ \\
\hline Arthralgia & $68(82.93)$ & $14(17.07)$ \\
Myalgia & $64(78.05)$ & $18(21.95)$ \\
Tiredness & $59(71.95)$ & $23(28.05)$ \\
Fever & $37(45.12)$ & $37(45.12)$ \\
Chills & $57(69.51)$ & $27(32.93)$ \\
Headache & $62(75.61)$ & $20(24.39)$ \\
Conjunctivitis & $64(78.05)$ & $18(21.95)$ \\
Nausea And Vomiting & $72(87.8)$ & $10(12.2)$ \\
Sore throat & $77(93.9)$ & $5(6.1)$ \\
Dry cough & $28(34.15)$ & $54(65.85)$ \\
Dyspnea & $15(18.29)$ & $67(81.71)$ \\
\hline
\end{tabular}

Table 2

Medical history of admitted patients

\begin{tabular}{lll}
\hline \multicolumn{1}{c}{ Risk factors } & No $n(\%)$ & Yes $n(\%)$ \\
\hline Obesity & $71(86.59)$ & $11(13.41)$ \\
Chronic lung disease & $79(96.34)$ & $3(3.66)$ \\
Renal failure & $77(93.9)$ & $5(6.1)$ \\
Liver dysfunction & $82(100)$ & $0(0)$ \\
Metabolic disorders & $82(100)$ & $0(0)$ \\
Thalassemia & $82(100)$ & $0(0)$ \\
Immunodeficiency & $82(100)$ & $0(0)$ \\
Autoimmune disease & $82(100)$ & $0(0)$ \\
Neurological disease & $77(93.9)$ & $5(6.1)$ \\
Gastrointestinal diseases & $80(97.56)$ & $2(2.44)$ \\
Cardiovascular diseases & $80(97.56)$ & $2(2.44)$ \\
Pregnancy & $82(100)$ & $0(0)$ \\
Smoking & $73(89.02)$ & $9(10.98)$ \\
Hypertension & $50(60.98)$ & $32(39.02)$ \\
Diabetes & $61(74.39)$ & $21(25.61)$ \\
\hline
\end{tabular}

\section{Results}

Clinical and demographic data of a total 82 patients were assessed. The median age (IQR) of the patients was $57.32(45.75,70)$ years. Female/male ratio was 36 (43.9\%)/46 (56.09\%). Among the admitted patients, 26 patients $(31.7 \%)$ were hospitalized in ICU, one of them expired. Sixty seven $(81.7 \%)$ of patients needed oxygen therapy. Two patients $(2.4 \%)$ needed mechanical ventilation. The median (IQR) of hospitalization was 7 (5, 9) days. All patients were discharged with good health conditions except for one patient who died. Table 1 shows the clinical manifestations of admitted patients. The most common clinical symptoms were dyspnea $(81.71 \%)$ and cough $(65.85 \%)$. Fever was detected in $37(45.12 \%)$ of patients.

Assessment of past medical history of admitted patients revealed hypertension and diabetes as the most frequent chronic disorder which affected 32 (39.02\%) and $21(25.61 \%)$ of admitted patients, respectively. Totally, $33(40.2 \%)$ of individuals have a history of chronic diseases. In addition, $13(15.85 \%)$ of individuals have 
Table 3

The results of Bayesian one sample test to examine paraclinical factors with normal values

\begin{tabular}{|c|c|c|c|c|c|}
\hline Variables & Median & IQR & $95 \% \mathrm{crI}$ & $P$-value & Normal range \\
\hline \multicolumn{6}{|c|}{ Total patients } \\
\hline WBC & 6230 & {$[4680,9130]$} & {$[6614,9609]$} & 0.013 & $4000-10000$ \\
\hline $\mathrm{RBC}$ & 4.7 & {$[4.1,5.13]$} & {$[4.51,4.8]$} & $<0.0001$ & $3.5-5.5$ \\
\hline $\mathrm{MCV}$ & 84 & {$[82.48,86.4]$} & {$[82.6,84.85]$} & $<0.0001$ & $80-100$ \\
\hline $\mathrm{MCH}$ & 27.5 & {$[26,28.28]$} & {$[26.55,27.5]$} & $<0.0001$ & $28-32$ \\
\hline $\mathrm{MCHC}$ & 32.1 & {$[31.25,33.11]$} & {$[32.01,32.54]$} & 0.035 & $32-36$ \\
\hline PLT & 195000 & {$[145000,263000]$} & {$[195495.9,232923.8]$} & $<0.0001$ & $150000-30000$ \\
\hline LYM & 21 & {$[12,29]$} & {$[18.91,23.21]$} & 0.324 & $20-40$ \\
\hline NEUT & 69 & {$[60,79]$} & {$[67,71.98]$} & 0.687 & $30-70$ \\
\hline IL-6 & 18.7 & {$[7.06,44.27]$} & {$[45.8,130.9]$} & 0.001 & $50-100$ \\
\hline CRP & 57.5 & {$[14.2,113]$} & {$[57.4,83.76]$} & $<0.0001$ & $<3$ \\
\hline \multicolumn{6}{|l|}{ Males } \\
\hline $\mathrm{HB}$ & 13.6 & {$[11.2,14.1]$} & {$[12.6,13.9]$} & 0.021 & $14-17$ \\
\hline HCT & 41 & {$[37.3,46.1]$} & {$[39.2,42.9]$} & 0.953 & $41-51 \%$ \\
\hline ESR & 35 & {$[18,60]$} & {$[32.8,50.39]$} & $<0.0001$ & $0-15$ \\
\hline \multicolumn{6}{|l|}{ Females } \\
\hline HB & 11.77 & {$[10.67,12.95]$} & {$[11.32,12.21]$} & 0.3 & $12-16$ \\
\hline HCT & 36.55 & {$[33.7,40.2]$} & {$[35.22,37.89]$} & 0.395 & $36-47 \%$ \\
\hline ESR & 48.85 & {$[20,68.5]$} & {$[38.5,59.2]$} & 0.0001 & $0-20$ \\
\hline
\end{tabular}

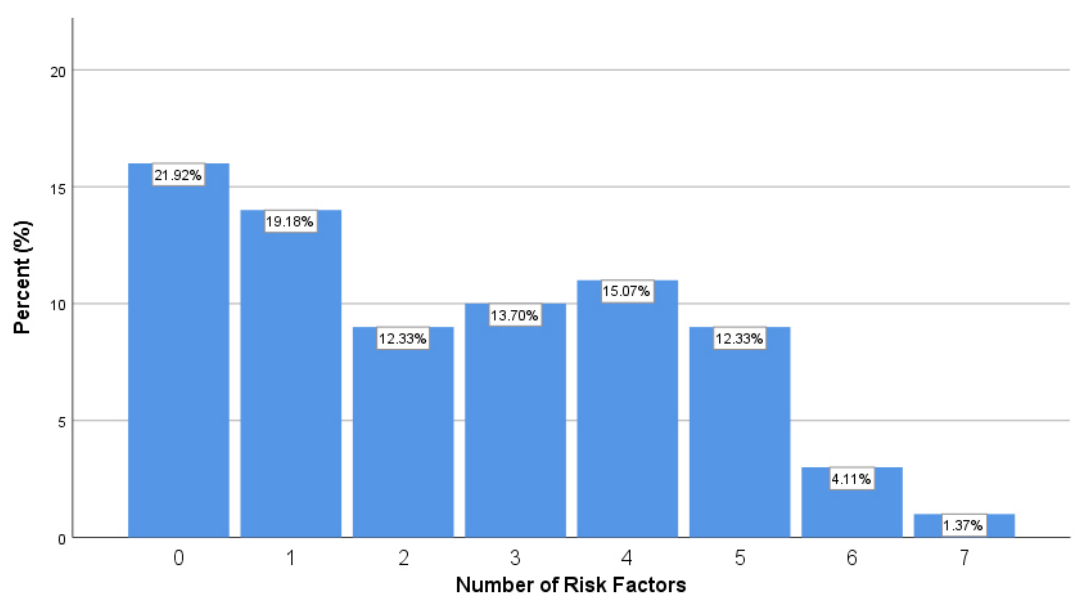

Fig. 1. Proportions of patients with certain numbers of risk factors.

family history of chronic diseases. Table 2 shows the summary of medical history of admitted patients.

Totally, $21.92 \%$ of patients had none of the mentioned risk factors, while $1.37 \%$ had seven risk factors. Figure 1 shows the proportion of patients with each mentioned risk factors.

A comprehensive laboratory investigation was performed at the time of admission. The median values, normal ranges of parameters, $95 \%$ confidence intervals and Interquartile (IQR) are shown in Table 3. Statistical analyses showed significant deviations from the normal range in the WBC count, RBC count, MCV, $\mathrm{MCH}, \mathrm{MCHC}$, Platelet count, CRP and IL-6 levels in the admitted patients.

Totally, $12(14.63 \%)$ and $14(17.07 \%)$ of patients had low and high WBC counts, respectively. Lymphopenia was detected in $36(43.9 \%)$ of patients, while $6(7.32 \%)$ of patients had lymphocytosis. High levels of Il-6 were detected in $4(4.88 \%)$ of patients. CRP levels were elevated in $69(84.1 \%)$ of patients. Table 4 shows the proportion of patients with lower/normal/upper values of laboratory parameters.

\subsection{Comparison between ICU-admitted patients and other patients}

The median age of patients who were admitted to ICU and the other patients were 63.56 and 54.27 years, respectively ( $P=0.047)$. Subsequently, we compared median values of laboratory parameters between patients admitted to ICU and other patients. CRP and IL-6 
Table 4

The number $(\%)$ of patients with lower/normal/upper values of laboratory parameters

\begin{tabular}{llllllllllllll}
\hline Category & WBC & RBC & MCV & MCH & MCHC & PLT & LYM & NEUT & IL-6 & CRP & HB & HCT & ESR \\
\hline Lower & 12 & 5 & 9 & 49 & 40 & 27 & 36 & 0 & 66 & - & 42 & 39 & - \\
& $(14.63)$ & $(6.1)$ & $(10.98)$ & $(59.76)$ & $(36.04)$ & $(32.93)$ & $(43.9)$ & & $(80.49)$ & $(51.22)$ & $(47.56)$ & & \\
Normal & 56 & 64 & 73 & 33 & 71 & 47 & 40 & 49 & 12 & 13 & 39 & 38 & 19 \\
& $(68.29)$ & $(78.05)$ & $(89.02)$ & $(40.24)$ & $(63.96)$ & $(57.32)$ & $(48.78)$ & $(59.76)$ & $(14.63)$ & $(15.8)$ & $(47.56)$ & $(46.34)$ & $(23.17)$ \\
Upper & 14 & 13 & 0 & 0 & 0 & 8 & 6 & 33 & 4 & 69 & 1 & 5 & 63 \\
& $(17.07)$ & $(15.85)$ & & & & $(9.76)$ & $(7.32)$ & $(40.24)$ & $(4.88)$ & $(84.1)$ & $(1.22)$ & $(6.1)$ & $(76.83)$ \\
\hline
\end{tabular}

Table 5

Comparison of laboratory and clinical findings between patients admitted to ICU and the other patients

\begin{tabular}{|c|c|c|c|c|c|}
\hline Factor & ICU & Median & [IQR] & Difference & $P$-value \\
\hline \multirow[t]{2}{*}{ Age } & Yes & 63.56 & {$[50.7,74.5]$} & 9.28 & 0.047 \\
\hline & No & 54.27 & {$[38,64]$} & & \\
\hline \multirow[t]{2}{*}{ CRP } & Yes & 95 & {$[43.35,125.5]$} & 68 & 0.002 \\
\hline & No & 27 & {$[5,78]$} & & \\
\hline \multirow[t]{2}{*}{ IL-6 } & Yes & 45 & {$[15.3,67.2]$} & 31 & 0.004 \\
\hline & No & 14 & {$[6.5,31.625]$} & & \\
\hline \multirow[t]{2}{*}{ ESR } & Yes & 50 & {$[33.5,59.5]$} & 18 & 0.099 \\
\hline & No & 32 & {$[18,54.5]$} & & \\
\hline \multirow[t]{2}{*}{ WBC } & Yes & 6350 & {$[5332.5,8240]$} & 720 & 0.279 \\
\hline & No & 5630 & {$[4260,8040]$} & & \\
\hline \multirow[t]{2}{*}{$\mathrm{RBC}$} & Yes & 5 & {$[4.1675,5.01]$} & 0.1545 & 0.441 \\
\hline & No & 5 & {$[4.13,5.3]$} & & \\
\hline \multirow[t]{2}{*}{$\mathrm{HB}$} & Yes & 12 & {$[10.975,13]$} & -0.892 & 0.126 \\
\hline & No & 13 & {$[11.7,14.5]$} & & \\
\hline \multirow[t]{2}{*}{$\mathrm{MCV}$} & Yes & 83 & {$[81.4475,86.3025]$} & -0.98 & 0.291 \\
\hline & No & 84 & {$[82.73,86]$} & & \\
\hline \multirow[t]{2}{*}{$\mathrm{MCH}$} & Yes & 27 & {$[25.6725,28.15]$} & -0.768 & 0.207 \\
\hline & No & 28 & {$[26.19,28.67]$} & & \\
\hline \multirow[t]{2}{*}{$\mathrm{MCHC}$} & Yes & 32 & {$[30.6,33]$} & -0.524 & 0.187 \\
\hline & No & 32 & {$[31.38,33.24]$} & & \\
\hline \multirow[t]{2}{*}{ HCT } & Yes & 37 & {$[35.575,40]$} & -1.99 & 0.26 \\
\hline & No & 39 & {$[34.6,45]$} & & \\
\hline \multirow[t]{2}{*}{ PLT } & Yes & 171000 & {$[137000,225750]$} & -5970 & 0.878 \\
\hline & No & 176000 & {$[128000,229000]$} & & \\
\hline \multirow[t]{2}{*}{ LYM } & Yes & 18 & {$[9,27.25]$} & -4.96 & 0.114 \\
\hline & No & 23 & {$[14,30]$} & & \\
\hline \multirow[t]{2}{*}{ NEUT } & Yes & 71 & {$[65.75,80.5]$} & 6.31 & 0.076 \\
\hline & No & 68 & {$[58,78]$} & & \\
\hline \multirow[t]{2}{*}{ Fever } & Yes & 37.5 & {$[37,38]$} & 0.5 & 0.505 \\
\hline & No & 37 & {$[37,38]$} & & \\
\hline
\end{tabular}

levels were higher in patients who were admitted to ICU ( $P$ values $=0.002$ and 0.004 , respectively). Other laboratory parameters were not significantly different between two groups. Table 5 shows the results of comparisons between these two groups.

\section{Discussion}

In the current study, we presented the clinical and demographic data of patients with COVID-19 who were admitted in a single hospital in Tehran. In our cohort of COVID-19 patients, we detected a higher number of male patients than female patients. Such sex bias has been previously reported in similar viral infections including MERS-CoV and SARS-CoV [10,11] and in SARS-CoV-2 case series from other population [12].

Disease course and clinical manifestations of COVID-19 have been assessed in different populations. A retrospective, multicenter cohort study in Wuhan, China has reported the presence of a comorbid condition in $48 \%$ of patients, with hypertension, diabetes and coronary heart disease being the most frequent comorbid conditions, respectively [3]. The results of the present single-center study are consistent with this study regarding the presence of comorbid conditions. Yet the percentage of affected individuals with these conditions was higher than the previously reported value. In ad- 
dition, the frequency of chronic underlying diseases in patients infected with SARS-Cov-2 is comparable with MERS-CoV [10].

Moreover, the frequencies of clinical manifestations such as dyspnea, cough, fever and myalgia in our cohort of patients are comparable with the recent single-center study by Ashraf et al. [8]. The prevalence of fever and cough in these two Iranian studies is comparable with the statistics reported in a cohort of Chinese patients including 1099 from 552 hospitals [13]. Yet, the frequency of fever at the admission is significantly lower from the reports of two single-center case series of 138 and 99 Chinese patients which detected fever in $98.6 \%$ and $83 \%$ of patients, respectively $[12,14]$.

In the present cohort of patients, $12.2 \%$ of patients had atypical symptoms, such as nausea and vomiting at the time of admission. Consistent with this finding, Wang et al. have reported diarrhea and nausea as initial symptoms in a significant percentage of patients [14]. Thus, special consideration should be given in assessment of suspected patients with these symptoms.

Totally, $12(14.63 \%)$ and 14 (17.07\%) of patients had low and high WBC counts, respectively. Lymphopenia was detected in $36(43.9 \%)$ of patients, while $6(7.32 \%)$ of patients had lymphocytosis. These data is not consistent with the results of Chen et al. study which reported decrease in the absolute number of lymphocytes in most COVID-19 patients. They suggested that SARS-CoV-2 may largely act on lymphocytes, particularly T lymphocytes, in a similar way to SARS-CoV [12]. They also suggested that the cytokine storm caused by the virus might cause a sequence of immune responses leading to alteration in the number of peripheral WBCs including lymphocytes [12]. The discrepancy between our results and the results of mentioned study might be due to small sample size of our study or ethnic factors. Another single-center study from Iran has reported higher WBC counts in critically ill patients compared with the other group of patients [8]. However, we only detected higher CRP and IL-6 levels in patients admitted to ICU compared with other patients. Assessment of data from other centers and meta-analysis of the whole clinical and laboratory findings would provide a more elaborate outlook of the clinical and paraclinical findings associated with COVID-19 [15].

We also assessed IL-6 levels in COVID-19 patients. High levels of IL-6 were detected in 4 (4.88\%) of patients. Moreover, the median level of this cytokine was higher in patients who were admitted to ICU. Consistent with this finding, elevation of in IL-6 levels in patients with COVID-19 has been associated with the severity of disorder [16]. Meanwhile, inhibition of IL-6 release has been suggested as an effective modality for treatment of COVID-19 [17].

In brief, the current study in a cohort of COVID-19 patients with confirmed molecular diagnosis shows the heterogeneous clinical manifestations and paraclinical parameters in these patients. Future assessment of clinical course and laboratory findings in larger cohorts of patients would facilitate identification of risk factors and predictive markers for COVID-19 severity.

\section{Acknowledgments}

The current study was supported by a grant from Shahid Beheshti University of Medical Sciences.

\section{References}

[1] W.H. Organization, Coronavirus disease 2019 (COVID-19): situation report, 2020, 80 .

[2] A.R. Tuite et al., Estimation of coronavirus disease 2019 (COVID-19) burden and potential for international dissemination of infection from Iran, Annals of Internal Medicine 172 (2020), 699-701.

[3] F. Zhou et al., Clinical course and risk factors for mortality of adult inpatients with COVID-19 in Wuhan, China: A retrospective cohort study, The lancet, 2020.

[4] S. Ghafouri-Fard et al., Effects of host genetic variations on response to, susceptibility and severity of respiratory infections, Biomedicine and Pharmacotherapy 128 (2020).

[5] S. Ghafouri-Fard et al., Angiotensin converting enzyme: A review on expression profile and its association with human disorders with special focus on SARS-CoV-2 infection, Vascular Pharmacology 130 (2020).

[6] G. Lippi, C. Mattiuzzi, F. Sanchis-Gomar and B.M. Henry, Clinical and demographic characteristics of patients dying from COVID-19 in Italy versus China, Journal of medical virology, 2020.

[7] K. Heydari et al., Clinical and paraclinical characteristics of COVID-19 patients: A systematic review and meta-analysis, medRxiv, 2020.

[8] M.A. Ashraf et al., COVID-19 in Iran, a comprehensive investigation from exposure to treatment outcomes, medRxiv, 2020.

[9] A. Ghaysouri et al., New clinical experiences and evaluation of clinical and paraclinical features of deceased patients with COVID-19 infection referred to Shahid Mostafa Khomeini Hospital of Ilam, Travel Med Infect Dis, 2020, 101657101657.

[10] A. Badawi and S.G. Ryoo, Prevalence of comorbidities in the middle east respiratory syndrome coronavirus (MERS-CoV): A systematic review and meta-analysis, International Journal of Infectious Diseases 49 (2016), 129-133.

[11] R. Channappanavar et al., Sex-based differences in susceptibility to severe acute respiratory syndrome coronavirus infection, The Journal of Immunology 198 (2017), 4046-4053. 
[12] N. Chen et al., Epidemiological and clinical characteristics of 99 cases of 2019 novel coronavirus pneumonia in Wuhan, China: A descriptive study, The Lancet 395 (2020), 507-513.

[13] W.J. Guan et al., Clinical characteristics of coronavirus disease 2019 in China, The New England Journal of Medicine 382 (2020), 1708-1720

[14] D. Wang et al., Clinical characteristics of 138 hospitalized patients with 2019 novel coronavirus-infected pneumonia in Wuhan, China, JAMA 323 (2020), 1061-1069.
[15] R. Noroozi et al., Altered cytokine levels and immune responses in patients with SARS-CoV-2 infection and related conditions, Cytokine 133 (2020).

[16] T. Herold et al., Level of IL-6 predicts respiratory failure in hospitalized symptomatic COVID-19 patients, medRxiv 2020 , Google Scholar, 2020.

[17] B. Liu, M. Li, Z. Zhou, X. Guan and Y. Xiang, Can we use interleukin-6 (IL-6) blockade for coronavirus disease 2019 (COVID-19)-induced cytokine release syndrome (CRS)? Journal of Autoimmunity, 2020, 102452. 